\title{
Scaling laws of diffusion and time intermittency generated by coherent structures in atmospheric turbulence
}

\author{
P. Paradisi ${ }^{1}$, R. Cesari ${ }^{2}$, A. Donateo ${ }^{2}$, D. Contini ${ }^{2}$, and P. Allegrini ${ }^{3,4}$ \\ ${ }^{1}$ Istituto di Scienza e Tecnologie dell'Informazione “A. Faedo" (ISTI-CNR), Via Moruzzi 1, 56124 Pisa, Italy \\ ${ }^{2}$ Istituto di Scienze dell'Atmosfera e del Clima, Lecce Unit, Strada Provinciale Lecce-Monteroni km. 1.200, \\ 73100 Lecce, Italy \\ ${ }^{3}$ Istituto di Fisiologia Clinica (IFC-CNR), Via Moruzzi 1, 56124 Pisa, Italy \\ ${ }^{4}$ Centro EXTREME, Scuola Superiore S. Anna, P.zza Martiri della Libertá 7, 56127 Pisa, Italy \\ Correspondence to: P. Paradisi (paolo.paradisi@isti.cnr.it)
}

Received: 23 June 2011 - Revised: 6 December 2011 - Accepted: 3 February 2012 - Published: 15 February 2012

\begin{abstract}
We investigate the time intermittency of turbulent transport associated with the birth-death of self-organized coherent structures in the atmospheric boundary layer. We apply a threshold analysis on the increments of turbulent fluctuations to extract sequences of rapid acceleration events, which is a marker of the transition between self-organized structures.

The inter-event time distributions show a power-law decay $\psi(\tau) \sim 1 / \tau^{\mu}$, with a strong dependence of the power-law index $\mu$ on the threshold.

A recently developed method based on the application of event-driven walking rules to generate different diffusion processes is applied to the experimental event sequences. At variance with the power-law index $\mu$ estimated from the inter-event time distributions, the diffusion scaling $H$, defined by $\left\langle X^{2}\right\rangle \sim t^{2 H}$, is independent from the threshold.

From the analysis of the diffusion scaling it can also be inferred the presence of different kind of events, i.e. genuinely transition events and spurious events, which all contribute to the diffusion process but over different time scales. The great advantage of event-driven diffusion lies in the ability of separating different regimes of the scaling $H$. In fact, the greatest $H$, corresponding to the most anomalous diffusion process, emerges in the long time range, whereas the smallest $H$ can be seen in the short time range if the time resolution of the data is sufficiently accurate.

The estimated diffusion scaling is also robust under the change of the definition of turbulent fluctuations and, under the assumption of statistically independent events, it corresponds to a self-similar point process with a well-defined power-law index $\mu_{D} \sim 2.1$, where $D$ denotes that $\mu_{D}$ is
\end{abstract}

derived from the diffusion scaling. We argue that this renewal point process can be associated to birth and death of coherent structures and to turbulent transport near the ground, where the contribution of turbulent coherent structures becomes dominant.

\section{Introduction}

In the region of atmosphere very near to the ground, known as Atmospheric Boundary Layer (ABL), the structure of turbulence is highly intermittent. This is mainly related to the direct interaction of the air masses with the ground, which causes the growing of dynamical instabilities resulting in violent bursts of fluid moving upward or downward, in between periods of relatively quasi-stable slow motion with variable duration times (Paw et al., 1992; Kaimal and Finnigan, 1994; Katul et al., 1997; Finnigan, 2000; Poggi and Katul, 2007). The upward motion is given by the ejection of slow fluid masses that suddenly move upward forming a swirling structure. The opposite phenomenon of fluid intrusion from above to the ground, known as sweep motion, is also observed. In other words, sudden fluid accelerations occur randomly in time and space alternating with periods of quasi-stable motions. During the stable periods, it is possible to identify regions of fluid elements establishing a coherent motion. These coherent fluid structures are characterized by self-organized motion, which can be identified through the space-time correlation structure. Thus, there is a sporadic emergence or "birth" of coherent structures from regions of uncorrelated fluid elements. The evolution of the 
coherent structures, which has a relatively long life-time, is quasi-stable or quasi-stationary. During this life-time there is a slow increase of instabilities and, at some time instant, the instability becomes so large that a quasi-instantaneous passage to an unstable condition yields the "death" of the structures. We denote these short-time, quasi-instantaneous, transitions as "critical events". These coherent structures of self-organized fluid flow are then characterized by a birthdeath process of cooperation.

The critical events, marking the transition among different coherent fluid structures, are thus the most important manifestation of the turbulent intermittency. As an example, an important source of intermittent behavior in the ABL is given by the wake production, which is particularly important in canopy flows (Finnigan, 2000; Cava and Katul, 2009). Ejection-sweep processes also arise above grassland sites and determine the greatest contribution to the turbulent Reynold stress. Cava and Katul (2009) refer to this kind of phenomenology as "external intermittency", being the large scales directly affected by the boundaries, such as ground, vegetation and buildings, which can be regarded as a sort of "external" factor. Mahrt (1989) claims that large scale intermittency, called "global intermittency" and involving scales of motion larger that the main coherent eddies of the energy cascade, is associated with events of downward heat fluxes and vertical mixing of pollutants in strongly stratified boundary layer. It is then clear that coherent structure intermittency gives a substantial contribution to turbulent transport (Mahrt, 1989; Katul et al., 1997; Mahrt, 2007).

This statistical description of turbulence based on critical events is recognized to be a complementary investigation tool with respect to other classical approaches, such as the Reynolds averaging procedure or the spectral description introduced by Taylor (Narasimha and Kailas, 1990; Narasimha et al., 2007; Bershadskii et al., 2004a,b; Sreenivasan and Bershadskii, 2006; Cava and Katul, 2009; Poggi and Katul, 2010). Adopting this view of sporadic turbulent transport we focus on the identification of critical events and on the statistical characterization of the time intermittency generated by them. Different methods for identifying critical events have been proposed in literature. For example, Bershadskii et al. (2004a), within a laboratory experiment of turbulent convection, focus their attention on the events associated with the occurrence of plumes, corresponding to an organized activity of convection and define the events as zero-crossings of the temperature fluctuations. Narasimha et al. (2007) proposed a method for describing turbulent fluxes by encoding the turbulence signals as a sequence of "flux events", whose starting and ending points are defined by a passage through given thresholds. Sreenivasan and Bershadskii (2006) recognized that two different contributions to turbulent intermittency can be distinguished: one related to the fluctuation in the signal amplitude and the other one to the local frequency of oscillations. They investigated this last feature through the clustering properties of a dichotomous version of the signal, i.e. a sequence of critical events defined as threshold passages and denoted as Telegraphic Approximation. Applying this approach to different data-sets, several authors determined the scaling exponents associated with clustering properties, intermittency and power spectrum, in some cases also finding functional relations among them (Bershadskii et al., 2004a,b; Sreenivasan and Bershadskii, 2006; Cava and Katul, 2009).

Herein we transform the turbulent velocity into a sequence of critical events. Having in mind the birth-death process of coherent structures, we select events corresponding to abrupt changes in the turbulent velocity, i.e. to the occurrence of rapid fluid accelerations. Our events are threshold passages of the signal increments, and not of the signal values as in Sreenivasan and Bershadskii (2006). The main idea is that this threshold passages should be a marker of the birth-death process of cooperation that we want to analyze. Paradisi et al. (2009a) used a similar definition and investigated the intermittent events generated by turbulence dynamics in the ABL, finding that the renewal hypothesis is satisfied with a good approximation. A sequence of events is defined to be a renewal point process when the inter-event times, or Waiting Times (WT), are mutually independent random variables (Cox, 1962; Lowen and Teich, 2005).

The renewal hypothesis is thought to be a basic feature of complex systems with sporadic randomness, i.e. displaying critical events with Non-Poisson statistics and power-law distributed WTs (Paradisi et al., 2005; Allegrini et al., 2006, 2007). We recall that Poisson statistics of the events means that, given a time interval $\left[t_{0}, t_{0}+t\right]$, the number of events $N(t)$ contained in this interval is distributed according to a Poisson law. In this case, the WT sequence is still renewal, but with exponential WT distribution, namely, there is a welldefined finite time scale.

It is important to note that the renewal assumption is also a fundamental hypothesis in the theoretical derivation of a new version of Linear Response Theory (LRT) and of a Fluctuation-Response Theorem based on Non-Poisson renewal events (Allegrini et al., 2007, 2011). Some theoretical predictions of this new LRT were also experimentally verified in weak turbulence regime of nematic liquid crystals (Silvestri et al., 2009; Allegrini et al., 2009b). Renewal assumption is also a crucial hypothesis for the development of theoretical models of anomalous diffusion based on fractional calculus (Mainardi and Paradisi, 2001; Gorenflo et al., 2002a), such as the fractional Fick's law (Paradisi et al., 2001a,b; Gorenflo et al., 2002b), which could explain the counter-gradient phenomenon observed in canopy flows (Kaimal and Finnigan, 1994; Finnigan, 2000). The renewal assumption in the ABL is also in agreement with models proposed in literature (Higbie, 1935; Katul et al., 2006), such as the surface renewal model (Perlmutter, 1961), where a renewal hypothesis is explicitly assumed. Considering the general phenomenology of turbulence and, in particular, the ground-turbulence interaction in the bottom part of the ABL, i.e. the Surface Layer, this seems to be a general feature of 
atmospheric turbulence. Then, following the cited literature, we assume a renewal hypothesis for the critical events. In the presence of renewal events, transport processes are dominated, in the long-time range, by the asymptotic properties of the corresponding WT distribution. This is certainly true if the selected events are exactly the renewal Non-Poisson events driving the system. This is not always true experimentally, and we expect to select spurious events and to disregard some of the complex ones related to self-organization. Nevertheless, the asymptotics of transport properties are still dominated by the long-time properties of the renewal NonPoisson events driving the system (Allegrini et al., 2006, 2007, 2010b).

Herein we investigate the self-similarity of the birth-death process driving the emergence of self-organization. To this goal, we firstly evaluate the scaling of two diffusion processes driven by the critical events previously detected from turbulent velocities in the ABL. In the renewal case, estimating the diffusion scaling allows also to evaluate the selfsimilarity index of the WT distribution $\psi(\tau)$, i.e. the exponent $\mu$ of the inverse power-law tail $\psi(\tau) \sim 1 / \tau^{\mu}$ (Allegrini et al., 2009a). This can be defined as a complexity measure of the underlying dynamics generating the renewal events, and it will be denoted in the following as complexity index (Allegrini et al., 2009a, 2010a). Complexity is here identified as the ability of the system to dynamically generate highly connected, self-organized structures. This is the case of the coherent fluid structures described above. The smaller is $\mu$ (but always above 1), the more complex the system is.

The paper is organized as follows. In Sect. 2 we introduce the method used to detect the events, which is then applied to experimental data of turbulence velocity in the ABL. In Sect. 3 we introduce renewal processes and two different random walks defining the event-driven diffusion processes. The relation between fractal dimension and complexity index is also discussed. In Sect. 4 we estimate the scaling of the diffusion processes by using the Detrended Fluctuation Analysis (DFA) (Peng et al., 1994). Finally, in Sect. 5 we discuss the results and draw some conclusions.

\section{Detecting critical events in turbulence}

In order to estimate diffusion scaling and self-similarity of time intermittency, both associated with the critical events, the statistical analysis was performed on experimental observations of turbulent velocities in the ABL. The data were collected in a measurement campaign carried out in Lecce during March 2004. The site was the experimental field inside the University Campus, about $3.5 \mathrm{~km}$ South-West of Lecce, in the Salentum Peninsula (Apulia region), South-East part of Italy (N40820010.800, E18807021.000 WGSA). The site is characterized by short vegetation, with two contiguous sides surrounded by small trees. There are a few sparse buildings in the surrounding area over an extension of about $1 \mathrm{~km}$. The nearest building is about $100 \mathrm{~m}$ far from the measurement site. Moreover, there could be possible wake effects on the measured turbulence. The roughness length is about $0.5 \mathrm{~m}$ and the displacement height about $7.5 \mathrm{~m}$. The instrumental set-up for the measurement of air velocity was an ultrasonic anemometer Gill R3, operating at a sampling frequency of $\omega=100 \mathrm{~Hz}$, mounted on a horizontal bar placed at the top of a telescopic mast, $9.6 \mathrm{~m}$ above the ground. The analysis is performed on time series about $8 \mathrm{~h}$ long.

The analysis is carried out on each velocity component independently from the other ones. As the scaling analysis is performed on a time segment whose duration is several hours, non-stationary trends are apparent in the velocity time series. In fact, the local mean wind depends on the largescale meteorological circulation patterns, such as the largescale pressure gradients, whose variability is on time scales of about $1 \mathrm{~h}$ (Holton, 1992). As we are interested in rapid acceleration associated with turbulent fluctuations, we follow the standard approach, used in boundary-layer meteorology, to define turbulent velocity fluctuations (Stull, 1988). Thus, the mean wind is estimated with the Moving Average (MA), defined by:

$\bar{S}(t)=\frac{1}{T} \int_{t-T / 2}^{t+T / 2} S\left(t^{\prime}\right) \mathrm{d} t^{\prime}=\frac{1}{N} \sum_{k=n-\frac{N}{2}}^{n+\frac{N}{2}-1} S(k \Delta t)$,

where $t=j \Delta t$ for some integer $j, \Delta t=1 / 100 \mathrm{~s}$ is the sampling time, $T$ the averaging time ( $30 \mathrm{~min}$ in our case), and $N=T / \Delta t$ the number of measured data in a time interval $T(N=180000$ in our case), and $S(t)$ is the generic turbulent signal, corresponding here to the three velocity components. After evaluating the mean wind with the Moving Average method, the turbulent fluctuations are computed as the difference between the total velocity and the mean wind. As known from similarity theory (Stull, 1988), the intensity of turbulent fluctuations, i.e. the turbulent kinetic energy, depends on the mean wind, which in turn depends on the large-scale circulation. This means that non-stationary effects could arise not only in the mean wind, but also in the turbulence intensity, and could affect the threshold analysis. Paradisi et al. (2009a) suggested that the modulation from the large-scale circulation, interpreted as an external perturbation acting on the local turbulence, could explain the slight deviations from the renewal condition. In general, the external perturbation of a renewal process was shown to be a delicate issue to be considered when estimating the selfsimilarity of the WT distribution and in the evaluation of renewal condition (Bianco et al., 2005b; Akin et al., 2006; Bianco et al., 2007; Akin et al., 2009; Paradisi et al., 2008, $2009 \mathrm{~b}$ ), thus suggesting the need for reducing as much as possible the effect of non-stationary external perturbations.

In order to minimize the effect of non-stationary trends of the turbulence intensity, associated with large scale circulation, on the threshold analysis, we estimate the local standard deviation $\sigma(k)$ : 


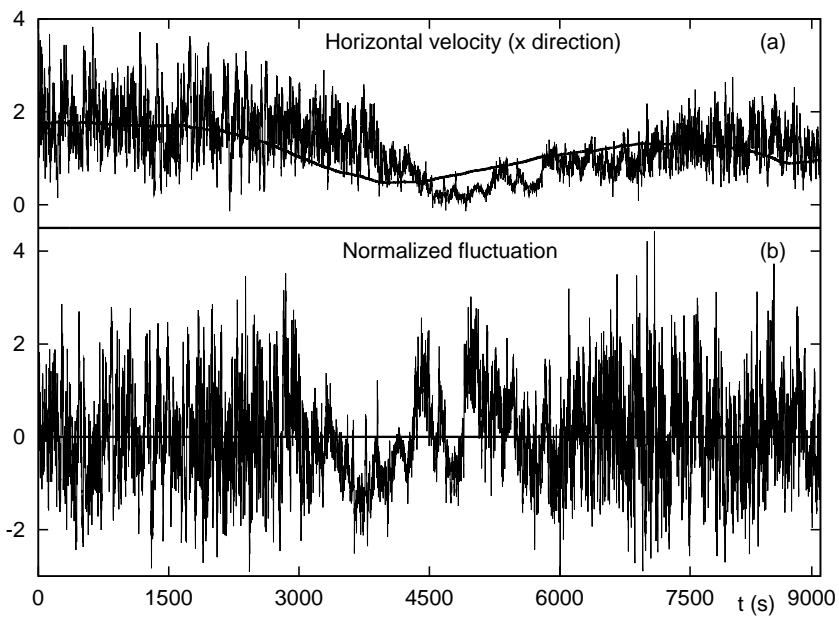

Fig. 1. Comparison of original signal and normalized fluctuation. (a) Sample from $U_{x}(t)$ time series and Moving Average $\bar{U}_{x}(t)$ (thick line). (b) normalized fluctuation $\widetilde{U}_{x}(t)$.

$$
\begin{aligned}
\sigma^{2}(k) & =\frac{1}{N} \sum_{m=k N+1}^{(k+1) N}\left(S_{m}-\bar{S}_{m}\right)^{2} ; \quad S_{m}=S(m \Delta t) ; \\
\bar{S}_{m} & =\bar{S}(m \Delta t),
\end{aligned}
$$

where the index $k$ labels the considered half-hour, being $k N \Delta t$ the beginning time of the half-hour $k$. Then, we define the normalized fluctuation:

$\widetilde{S}(t)=\frac{S(t)-\bar{S}(t)}{\sigma(k)}$,

where $k$ is such that: $k N \Delta t<t<(k+1) N \Delta t$.

In Fig. 1 we show a sample of the velocity $U_{x}(t)$ time series (panel a), compared with the normalized fluctuations $\widetilde{U}_{x}(t)$ (panel b), obtained by applying Eq. (3) to $U_{x}(t)$. In panel (a) the Moving Average is also reported.

The detection of critical events is done on the normalized fluctuations by marking the passage through a threshold $D_{0}$ of the fluctuation increments:

$\left|\Delta S_{m}\right|=|\widetilde{S}((m+1) \Delta t)-\tilde{S}(m \Delta t)|>D_{0}$

We note that, apart from the normalizing factor $\sigma(k)$, the statistical moments of $\left|\Delta S_{m}\right|$ correspond to the structure functions used to perform multi-scaling analysis (Frish, 1995). Structure functions are related to the energy flux across scales of motion. In fact, the structure function of order 2 can be associated with the turbulent energy dissipation $\epsilon$ in the inertial range by means of dynamical and similarity arguments. Then, it could be argued that sudden transitions in the turbulent fluctuation increments mirror abrupt transitions in the energy flux $\epsilon$. However, the feature we are investigating here is related to the more immediate kinematic interpretation of $\left|\Delta S_{m}\right|$ as a measure of fluid acceleration, so that the threshold passages in $\left|\Delta S_{m}\right|$ clearly correspond to rapid acceleration of the fluid masses. This is a (a) signal increments

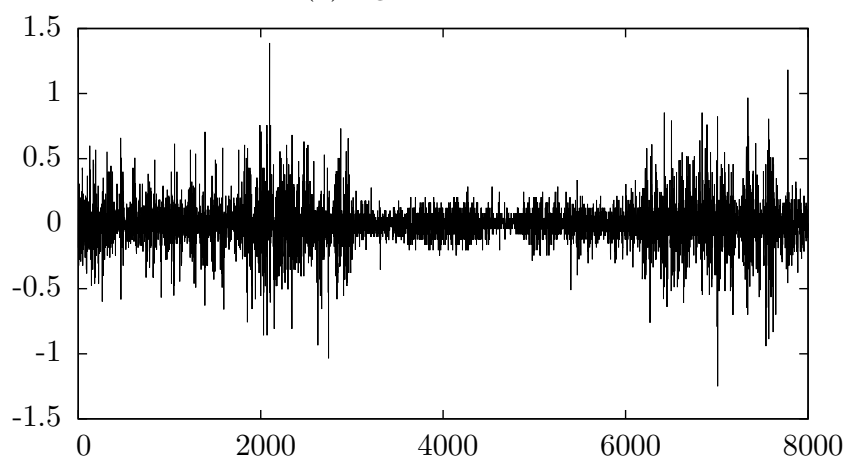

(b) WT sequence

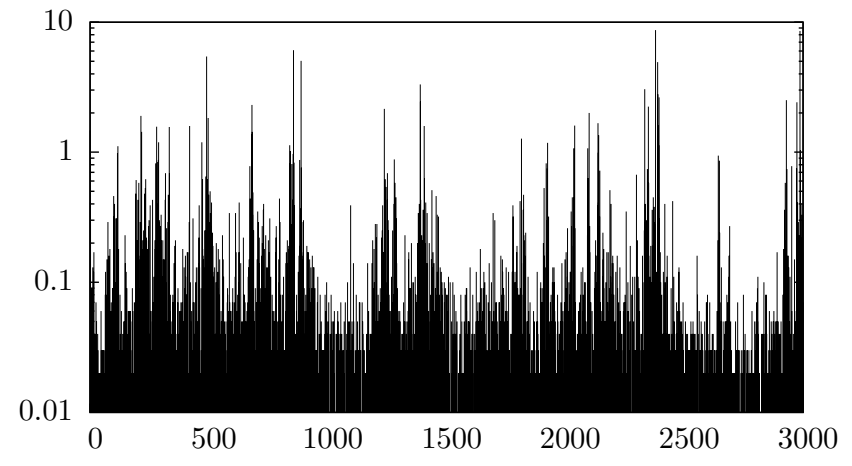

Fig. 2. Samples from (a) time series of increments of the normalized fluctuation $\widetilde{U}_{x}(t)$; (b) sequence of Waiting Times for threshold passages of $\Delta S=\Delta U_{x}\left(D_{0}=0.3\right)$.

natural choice to characterize the bursting behavior of sweepejection events related to birth-death of coherent structures.

When condition given by Eq. (4) is satisfied for the first time, the corresponding time $t_{1}=m_{1} * \Delta t$ is labeled as an event occurrence time. The procedure runs iteratively and the sequence $\left\{t_{n}=m_{n} \Delta t ; n=1,2, \ldots\right\}$ is obtained. The sequence of WTs $\left\{\tau_{n}\right\}$ is derived by the relation: $\tau_{n}=t_{n}-t_{n-1}$, $n=1,2, \ldots$ In general, the first time $t_{0}=0$ is not a time corresponding to an event, so that the first time $\tau_{1}$ is not a real WT and it can be disregarded. In any case, its effect on the scaling analysis is negligible.

The signal increments $\Delta U_{x}$, corresponding to the normalized fluctuations of Fig. 1b, are reported in Fig. 2a.

The critical events are detected from each normalized fluctuating velocity component for different thresholds: $D_{0}=$ $0.02,0.04,0.09,0.13,0.2,0.3$. For each velocity component and for each threshold, the sequences of occurrence times $t_{n}$ and WTs $\left\{\tau_{n}\right\}$ was derived. A sample of the associated sequence of WTs is reported in Fig. 2b for the threshold $D_{0}=0.3$. 


\section{Renewal processes and event-driven random walks}

As said in the Introduction, we follow the hypothesis that the critical events are renewal. In the following we give some definitions used in the theory of renewal processes (Cox, 1962). Then, we briefly discuss the fractal dimension of renewal point processes and, finally, we review the concept of event-driven diffusion, based on the model known as Continuous Time Random Walk (CTRW) (Montroll, 1964; Kenkre et al., 1973; Weiss and Rubin, 1983). The diffusion scaling of the event-driven random walk can be investigated to characterize not only the transport properties of turbulence in the $\mathrm{ABL}$, but also the complexity of the system. In fact, when the renewal hypothesis applies, it is possible to guess the complexity index $\mu$, as it will be explained below. The value of $\mu$ could be estimated through a direct power-law fit of the WT histogram, but it was shown that, when a difference among the two estimates is found, the diffusion scaling approach is more robust and reliable, since spurious events can affect the WT distribution (Allegrini et al., 2010b).

\subsection{Renewal processes}

A point process is defined as a series of events occurring randomly in time. This is described as a sequence of increasing random times: $\left\{t_{n}\right\}, n=0,1,2, \ldots, t_{n+1}>t_{n}$. The time instant $t_{0}=0$ is the time of the first event occurrence. Denoting the WTs by $\tau_{n}=t_{n}-t_{n-1}, n=1,2, \ldots$, the point process is defined to be a renewal process if the WTs $\tau_{n}$ are mutually independent random variables (Cox, 1962).

Under this assumption, the occurrence of an event is associated with a dynamical instability determining the basic property of erasing memory of the past. An example is given by the Manneville map (Manneville, 1980), displaying long-time "laminar" states with a small Lyapounov exponent and rapid transitions to short-time, quasi-instantaneous, "chaotic" states with a large Lyapounov exponent. The chaotic state, displaying a typical bursting behavior, has low predictability, so that a decay of memory is associated with the passage through this state, which can be seen as a quasiinstantaneous event marking the transition between two statistically independent laminar states.

In the time-homogeneous case, a renewal process is uniquely defined by a time-homogeneous Probability Density Function of WTs (WT-PDF): $\psi(\tau)$. Equivalently, it can be described by the local rate of event production $r(t)$, which is the expected number of events per time unit in a neighborhood of the time $t$ (Cox, 1962). Assuming that the last event occurred at $t_{n}$, the local rate $r(t)$ is the conditional probability density that an event occurs in an infinitesimal time interval $[t, t+\mathrm{d} t]$, given that no events occur in the time interval $\left[t_{n}, t\right]$ :

$r(t)=\lim _{\mathrm{d} t \rightarrow 0} \frac{1}{\mathrm{~d} t} \operatorname{Pr}\left\{t<t_{n+1} \leq t+\mathrm{d} t \mid t_{n+1}>t\right\}$.
By using the properties of conditional probabilities, it is possible to prove the following expression for the rate $r(t)$ (Cox, 1962):

$r(t)=\frac{\psi\left(t-t_{n}\right)}{\Psi\left(t-t_{n}\right)}=-\frac{1}{\Psi\left(t-t_{n}\right)} \frac{d \Psi}{\mathrm{d} t}\left(t-t_{n}\right) ; \quad t_{n}<t<t_{n+1}$,

where:

$\Psi(\tau)=\int_{\tau}^{\infty} \psi(s) d s=1-\int_{0}^{\tau} \psi(s) d s$

is the associated Survival Probability Function (WT-SPF), i.e. the probability that a WT $\tau_{j}$, extracted randomly from the ensemble of the WTs $\left\{\tau_{n}\right\}$, is larger than $\tau . r(t)$ is the instantaneous rate, which assumes a maximum value at an event, then it decays in time and, finally, it is reset to the maximum value at the next event, and so on. It is then possible to define an average decay rate $g(\tau)$, where $\tau$ is the time distance from the preceding event:

$g(\tau)=\frac{\psi(\tau)}{\Psi(\tau)}=-\frac{1}{\Psi(\tau)} \frac{d \Psi}{\mathrm{d} t}(\tau)$

Clearly, it results: $\psi(\tau)=-d \Psi(\tau) / d \tau$. It is worth noting that the renewal condition is given by the independence of $\psi(\tau)$ (and $\Psi(\tau)$ ) from the absolute time $t$, the index $n$ labeling the $n$-th event and the previous WTs (or, equivalently, the previous occurrence times). This is also seen in Eq. (6), defining the rate in the absolute time $t$ associated with the renewal sequence $\left\{t_{n}\right\}$. Only the explicit $t-t_{n}$ dependence can be seen, that is, the time elapsed from the last renewal event.

Once the rate function $g(\tau)$ is known, the WT-SPF is simply derived by solving Eq. (8) with respect to $\Psi(\tau)$ and imposing the initial condition $\Psi(0)=1$ :

$\Psi(\tau)=\exp \left(-\int_{0}^{\tau} g\left(\tau^{\prime}\right) d \tau^{\prime}\right)$.

The WT-SPF of a time-homogeneous Poisson process is an exponential function: $\Psi(\tau)=\exp \left(-r_{0} \tau\right)$; so that, comparing with Eq. (9), it results that the event rate is constant in time: $r(t)=r_{0}($ Cox, 1962). This is in agreement with the wellknown property that, in a Poisson process, the mean number of events in a given time interval $[t, t+\Delta t]$ is proportional to the length of the time interval itself: $\langle N[t, t+\Delta t]\rangle=r_{0} \Delta t$. At variance with Poisson processes, a renewal Non-Poisson process is characterized by a Non-Poisson distribution of the events, a non-exponential WT distribution and a changing in time event rate $g(t)$. An important example is given by the following rate:

$g(\tau)=\frac{r_{0}}{1+r_{1} \tau}$.

By applying Eq. (9), it is easy to prove the following expression for the WT-SPF: 
$\Psi(\tau)=\left(\frac{T_{0}}{T_{0}+\tau}\right)^{\mu-1} ; \quad T_{0}=\frac{1}{r_{1}} ; \quad \mu=1+\frac{r_{0}}{r_{1}}$

corresponding to the WT-PDF:

$\psi(\tau)=\frac{\mu-1}{T_{0}}\left(\frac{T_{0}}{T_{0}+\tau}\right)^{\mu}$.

These last two expressions become an inverse power-law, $1 / \tau^{\mu-1}$ and $1 / \tau^{\mu}$ respectively, in the long-time range $t \gg T_{0}$, thus satisfying scale invariance and self-similarity.

\subsection{Fractal dimension of renewal events}

Time intermittency is represented as a sequence of events occurring randomly in time, whose geometrical representation is given by a set of points on the time axis. As a consequence, it is possible to define the fractal dimension of the set composed by all the event occurrence times. This is done through the standard box counting method (Ott, 2002):

$N_{\mathrm{b}}\left(\Delta t_{\mathrm{b}}\right) \sim\left(\Delta t_{\mathrm{b}}\right)^{-D_{\mathrm{F}}}$

where $N_{\mathrm{b}}$ is the number of "boxes" (time intervals in our case) that contain at least one event. The algorithm is as follows. The event sequence is completely covered with a set of non-overlapping time intervals of size $\Delta t_{\mathrm{b}}$ (i.e. the length of the time intervals). Then, the number of interval containing at least one event is computed.

It is known that, for $\mu \leq 2$, the fractal dimension $D_{\mathrm{F}}$ is related to $\mu$ itself through the relationship:

$\mu=1+D_{\mathrm{F}}$.

This expression was rigorously derived by Lowen and Teich (2005) (see also Lowen and Teich, 1993) using exact analytical results of probability theory (Feller, 1971). A heuristic proof can be found in Schmitt et al. (1998) in the modeling of rainfall time series. Notice that Eq. (14) is valid only for Non-Poisson renewal point processes with $\mu \leq 2$, as it is evident by considering the geometrical limit $D_{\mathrm{F}} \leq 1$. For example, Schmitt et al. (1998) found this relationship to be experimentally verified only for the WT distribution of nonrainy periods but not for that of rainy periods.

In Fig. 3a we reported the results of the box counting algorithm applied to Monte-Carlo numerical simulations of the Non-Poisson renewal process described by the WT-PDF of Eq. (12). Two values of $\mu$ smaller than 2 and two greater than 2 were used. As expected, Eq. (14) is satisfied in the range $\mu<2$, which can be seen also as a testing on the quality of our numerical codes. On the contrary, the application of the box counting to the cases $\mu>2$ showed a dimension $D_{\mathrm{F}}=1$ independently from the value of $\mu$. The scaling is reached for time scales larger than the mean WT, which, at variance with the case $\mu<2$, assumes a finite value for $\mu>2$. In Fig. $3 b$ the same box counting analysis is applied to the experimental sequence of events derived from the turbulence data. The (a) WT-PDF

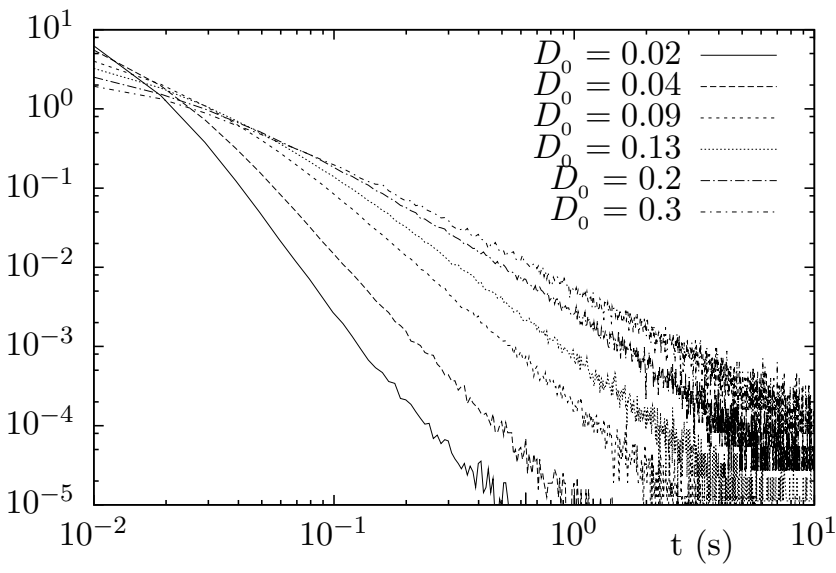

(b) WT-SPF

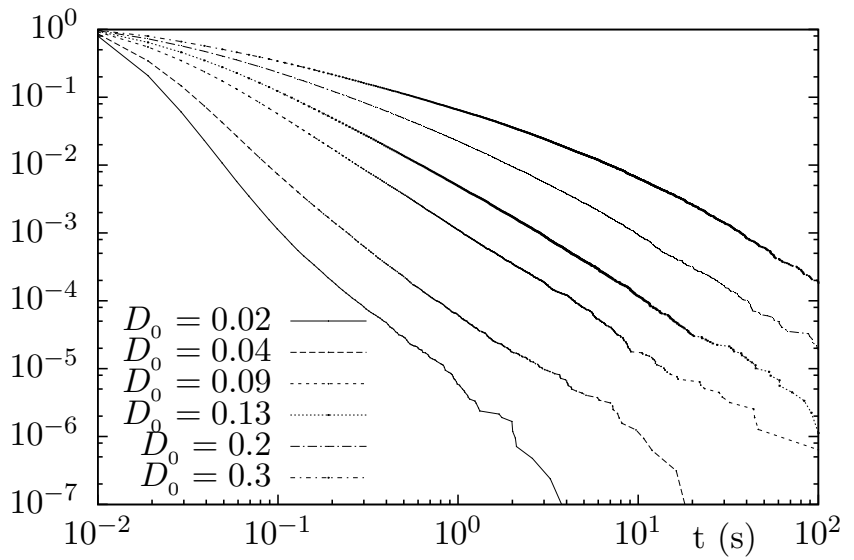

Fig. 3. Computation of the fractal dimension $D_{\mathrm{F}}$ through the box counting method. (a) Renewal model given by Eqs. (11)-(12), $T_{0}=$ 1 ; (b) turbulence data.

results are shown for different thresholds. The limiting behavior for times much larger than the average WT is always associated with a constant dimension $D_{\mathrm{F}}=1$, whereas in the short time range no evident power-law is observed. Consequently, we guess that short time range depends only on the average WT and, thus, on the threshold value.

It is interesting to compare these results with those obtained from the WT distribution. In Fig. 4 the probability density (WT-PDF) and survival probability (WT-SPF) of the WTs are reported for the $U_{x}$ component and for different threshold values. The WT distributions display a self-similar behavior, i.e. a power-law decay. As discussed in the introduction, this is a signature of complex behavior, which is the ability of the system to generate self-organization. By visual inspection of Fig. 4 it is evident that the complexity index $\mu$ evaluated from the WT distribution has a strong dependence on the threshold $D_{0}$.

In Table 1 the estimated values of $\mu$ are reported with the associated values of the threshold. It can be seen that the values span from the case of finite values to the case of infinite 
(a) WT-PDF

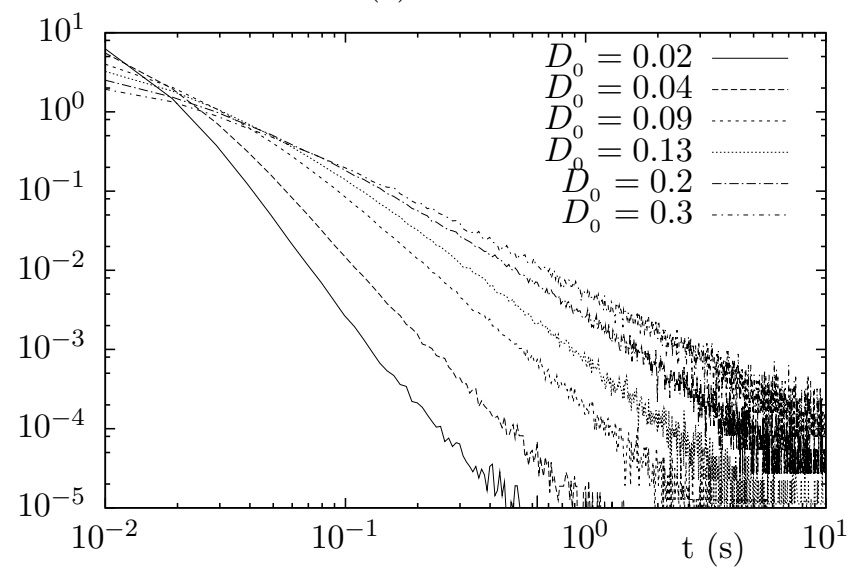

(b) WT-SPF

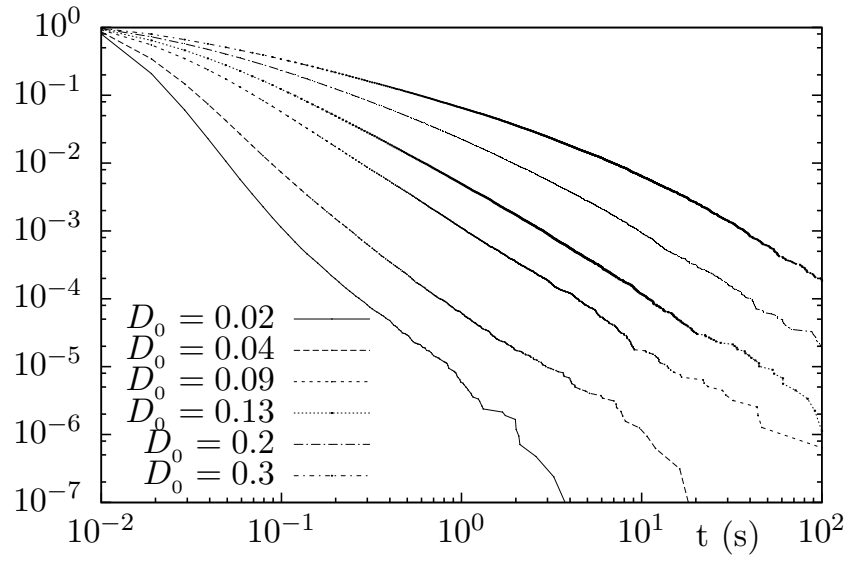

Fig. 4. Distribution of Waiting Times ( $U_{x}$ component): (a) probability density; (b) survival probability.

values of the average WT ( $\mu>2$ and $\mu \leq 2$, respectively) and of the variance ( $\mu>3$ and $\mu \leq 3$, respectively).

Further, it is worth noting that the higher threshold value, $D_{0}=0.3$, leads to a value $\mu=1.7$ (see Table 1), which is smaller than 2. According to Eq. (14), this should lead to a fractal dimension $D_{\mathrm{F}}=0.7$. On the contrary, after a short transient whose duration is about $1 \mathrm{~min}$, it again results $D_{\mathrm{F}}=1$. This is an indication that the estimation of $\mu$ from the WT distribution is probably not reliable, a condition that could be related to several causes, such as, for example, to the presence of spurious events not related to the selforganized structures. Even if large errors could occur in the direct estimation of $\mu$ from the WT distribution, a threshold dependence exists and can be seen as a manifestation of the multi-scaling and multi-fractal nature of turbulence, which is mainly associated with the intensity of the turbulent field (Schertzer and Lovejoy, 1987; Schertzer et al., 2002).

However, we are here more interested to investigate the role of intermittent time structure of self-organized coherent fluid motion in the scaling of turbulent transport. As
Table 1. Values of $\mu$ estimated from the WT distribution and the associated threshold values ( $U_{x}$ component).

\begin{tabular}{ccccccc}
\hline$D_{0}$ & 0.02 & 0.04 & 0.09 & 0.13 & 0.2 & 0.3 \\
\hline$\mu$ (from WT-PDF) & 4 & 3.3 & 2.7 & 2.4 & 2.1 & 1.7 \\
\hline
\end{tabular}

explained in the next Sections, this is done by investigating the scaling properties of diffusion generated by the sequences of critical events that define the birth-death process of cooperation.

The fact that we get always $D_{\mathrm{F}}=1$ means that the fractal analysis always detects a time homogeneous distribution of events for time longer than the average WT, which becomes finite for $\mu>2$ and is given by $\langle\tau\rangle=T_{0} /(\mu-2)$ in the case of renewal processes. However, even if $D_{\mathrm{F}}$ remains constant and equal to its limit value 1, a self-similarity is still observed in the event sequence through the complexity index $\mu$, which can then be exploited for the analysis of a more extended range of different conditions. This is seen by the WT distributions of Fig. 4 and will be confirmed by the diffusion scaling analysis in the next Sections.

\subsection{Event-driven random walks}

The CTRW model was first investigated by Montroll and coworkers (Montroll, 1964; Kenkre et al., 1973; Weiss and Rubin, 1983). At variance with the standard Random Walk with fixed time steps, it is allowed to have random time steps, in our case corresponding to sequence of renewal WTs $\left\{\tau_{n}\right\}$ extracted from the experimental time series. Here we want to study that part of the turbulent transport properties that are related to the time intermittency of critical events. To this goal, we investigate two different CTRWs driven by the same critical events, by defining two walking rules. Allegrini et al. (2009a) used this approach not only to estimate the complexity index $\mu$, but also to evaluate the renewal condition on a data-set of human brain ElectroEncephaloGrams.

The random walk is defined through a signal $\xi(t)$, which is a kind of random discontinuous velocity, taking two or three different values depending on the event occurrence times. This signal is highly discontinuous in time and intensity, as it jumps among two or three values and only at time instants randomly distributed in time. Thus, the diffusion variable is defined as:

$X(t)=X_{0}+\sum_{j=0}^{j=t} \xi(j) \Delta t$,

being $\Delta t$ the sampling time of the experimental time series. It is worth noting that the Taylor-Green-Kubo formula, relating the mean square displacement to the auto-correlation function, follows from this kinematic relationship. However, in this case, the classical version based on the stationary autocorrelation function (Taylor, 1921) must be modified to take 
(a) Asymmetric Jump (AJ)

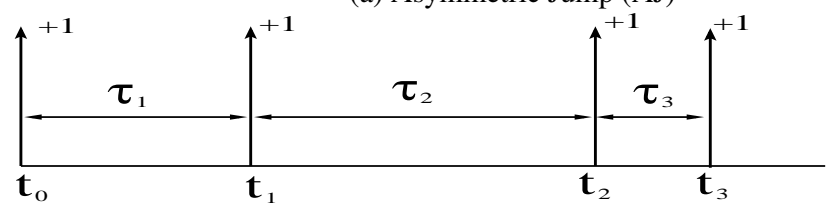

(b) Symmetric Jump (SJ)

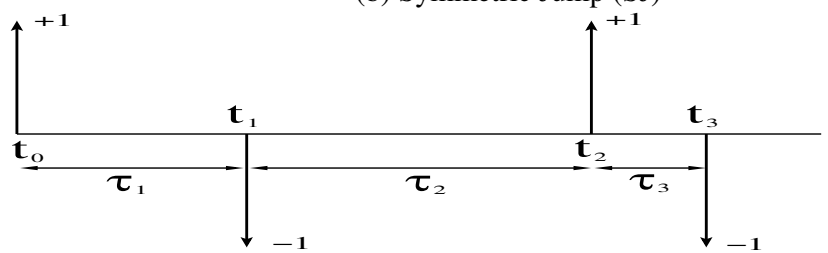

Fig. 5. The AJ and SJ walking rules for the "velocity signal" $\xi(t)$.

into account the lack of an invariant measure, i.e. of an equilibrium state in the long-time regime (Korabel et al., 2007). In other words, a stationary condition does not exist ans this is theoretically associated with the strong memory of the initial conditions. In many real systems with power-law behavior, such as turbulence in the ABL, a stationary condition can also exist. However, the relaxation to this stationary state can be so slow that the time scale of non-stationary trends, corresponding to the time scale over which the local (in time and space) equilibrium distribution changes, could be comparable or even shorter that the relaxation time of the local turbulence. The main consequence is that only anomalous diffusion is seen and the normal diffusion regime is never reached.

In Fig. 5 a sketch of the two signals $\xi(t)$ is reported. The times $t_{0}, t_{1}, t_{2}, \ldots$ correspond to the occurrence of the events $0,1,2, \ldots$, while $\tau_{1}, \tau_{2}, \ldots$ are the WTs, i.e. the time interval between the events 0 and 1 , the events 1 and 2 and so on. In particular, we have:

a. Asymmetric Jump (AJ) rule:

the walker makes a positive jump $\left(\xi\left(t_{n}\right)=1\right)$ in correspondence of each event $n$, otherwise it stands $(\xi(t)=$ $0)$. Then, $\xi(t)$ is a sequence of pulses of constant intensity.

b. Symmetric Jump (SJ) rule:

as in the AJ rule, but the walker can make positive or negative jumps in correspondence of an event: $\xi\left(t_{n}\right)=$ \pm 1 . The sign \pm is chosen with a coin tossing prescription.

The scaling properties of these random walks were extensively investigated in several papers (Shlesinger, 1974; Metzler and Klafter, 2000; Grigolini et al., 2001, 2002) by applying the analytical methods of CTRW model, i.e. the Montroll-Weiss equation for the Fourier-Laplace transform of the probability distribution $p(x, t) d x=\operatorname{Pr}[x<X(t) \leq x+$

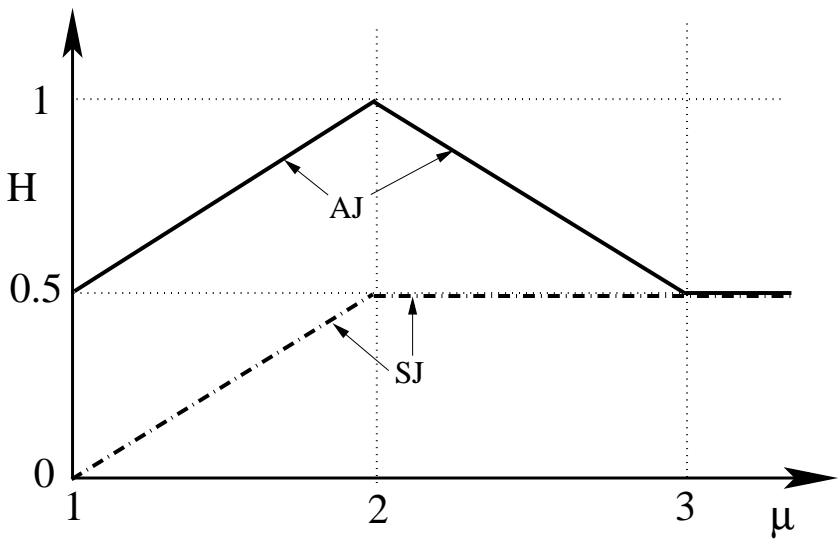

Fig. 6. Diffusion scaling $H$ vs. complexity index $\mu$ for SJ and AJ walking rules: AJ (continuous line), SJ (dotted-dashed line).

$d x$ ] (Montroll, 1964; Weiss and Rubin, 1983), or by applying simple scaling relationships in the case of AJ rule (Lowen and Teich, 1993; Allegrini et al., 2009a). In particular, two scaling indices were found in the asymptotic long-time limit: the self-similarity index $\delta$ of the diffusion probability distribution, defined by $p(x, t)=f\left(x / t^{\delta}\right) / t^{\delta}$; and the scaling exponent $H$ of the second moment. These scaling indices were determined in the case of Non-Poisson renewal WTs with inverse power-law tail: $\psi(\tau) \sim 1 / \tau^{\mu}$. Here the diffusion scaling will be investigated through the estimation of the second moment scaling exponent $H$, defined by:

$\sigma^{2}(t)=\left\langle(X(t)-\bar{X})^{2}\right\rangle \sim t^{2 H}$,

where $\bar{X}$ is the mean value of $X(t)$.

The analytical expressions for the diffusion scaling exponent $H$ as a function of the complexity index $\mu$ are plotted in Fig. 6 and summarized in the following:

AJ. $H= \begin{cases}\mu / 2 ; & 1<\mu<2 \\ 2-\mu / 2 ; & 2 \leq \mu<3 \\ 1 / 2 ; & \mu \geq 3\end{cases}$

SJ. $H= \begin{cases}(\mu-1) / 2 ; & 1<\mu<2 \\ 1 / 2 ; & \mu \geq 2\end{cases}$

Both rules give a normal scaling $H=1 / 2$ for $\mu \geq 3$, corresponding to normal (Gaussian) diffusion. For the SJ rule this is true also in the range $2<\mu \leq 3$, while AJ rule is superdiffusive $(H>1 / 2)$ in all the interval $1<\mu<3$. On the contrary, the $\mathrm{SJ}$ rule is sub-diffusive $(H<1 / 2)$ for $1<\mu<2$. We note that, if the WTs comes from a Poisson process, the value of $H$ is again $1 / 2$ and, in the long-time, we have a Gaussian diffusion.

The joint use of these walking rules can be used to evaluate the value of the $\mu$ by inverting the expressions given in Eqs. (17)-(18). Grigolini et al. (2001) found that the AJ 
rule is faster in reaching the scaling regime with respect to the other ones. Considering the finite length of experimental WT sequences, this means that the estimation of $\mu$ is usually more accurate in the AJ rule. It can be seen from Fig. 6 that $H_{\mathrm{AJ}}(\mu)$ is not an invertible function, as the same value of $H$ corresponds to two distinct values of $\mu$, one smaller and the other greater than 2. When $H_{\mathrm{SJ}}<1 / 2$ it results $\mu<2$ and both rules, i.e. the associated values of $\mu$ derived from AJ and SJ rules, could be compared to each other. On the contrary, for $H_{\mathrm{SJ}}=1 / 2$, a value of $\mu$ cannot be derived from the SJ rule, but we can assume $\mu>2$. For this reason, the SJ rule could be used to discriminate between $\mu<2$ and $\mu>2$, overcoming the ambiguity of $\mathrm{AJ}$ rule.

\section{Diffusion scaling}

Two diffusion processes are computed from the WT sequences extracted in Sect. 2. This is done by applying the AJ and SJ walking rules introduced in Sect. 3. Finally, the diffusion scaling $H$ of the two processes is estimated for each component independently from the other components.

The diffusion scaling $H$ of the two random walks is estimated by means of Detrended Fluctuation Analysis (DFA) (Peng et al., 1994). We briefly recall the main steps of this method:

- For a discrete time $L=4,5, \ldots$, the time series of the diffusion process $X(t)$ is split into not-overlapping time windows of length $L:[k L+1, k L+L]$. The window number is given by $[M / L]$, i.e. the integer part of $M / L$, being $M$ the total length of the time series.

- For each time window $[k L+1, k L+L] \quad(k=$ $0,1, \ldots,[M / L])$, the local trend is evaluated with a leastsquares straight line fit:

$\bar{X}_{k, L}(t)=a_{k, L} t+b_{k, L} ; k L<t \leq(k+1) L$.

- The fluctuation is derived in the usual way:

$$
\begin{aligned}
& \widetilde{X}_{k, L}(t)=X(t)-\bar{X}_{k, L}(t)= \\
& =X(t)-a_{k, L} t-b_{k, L} ; k L<t \leq(k+1) L .
\end{aligned}
$$

- For a given time scale $L$, the mean-square deviation of the fluctuation is calculated over every window:

$$
\begin{aligned}
& F^{2}(k, L)=\frac{1}{L} \sum_{t=k L+1}^{(k+1) L} \widetilde{X}_{k, L}^{2}(t)= \\
& =\frac{1}{L} \sum_{t=k L+1}^{(k+1) L}\left(X(t)-\bar{X}_{k, L}(t)\right)^{2}
\end{aligned}
$$

- Finally, an average over the windows is performed:

$$
F^{2}(L)=\frac{1}{[M / L]} \sum_{k=0}^{[M / L]} F^{2}(k, L)
$$

In the case of a self-similar process, it results: $F(L) \sim L^{H}$. Then, by defining $z=\log (F(L))$ and $y=\log (L)$, it is possible to apply a least-squares straight line fit:
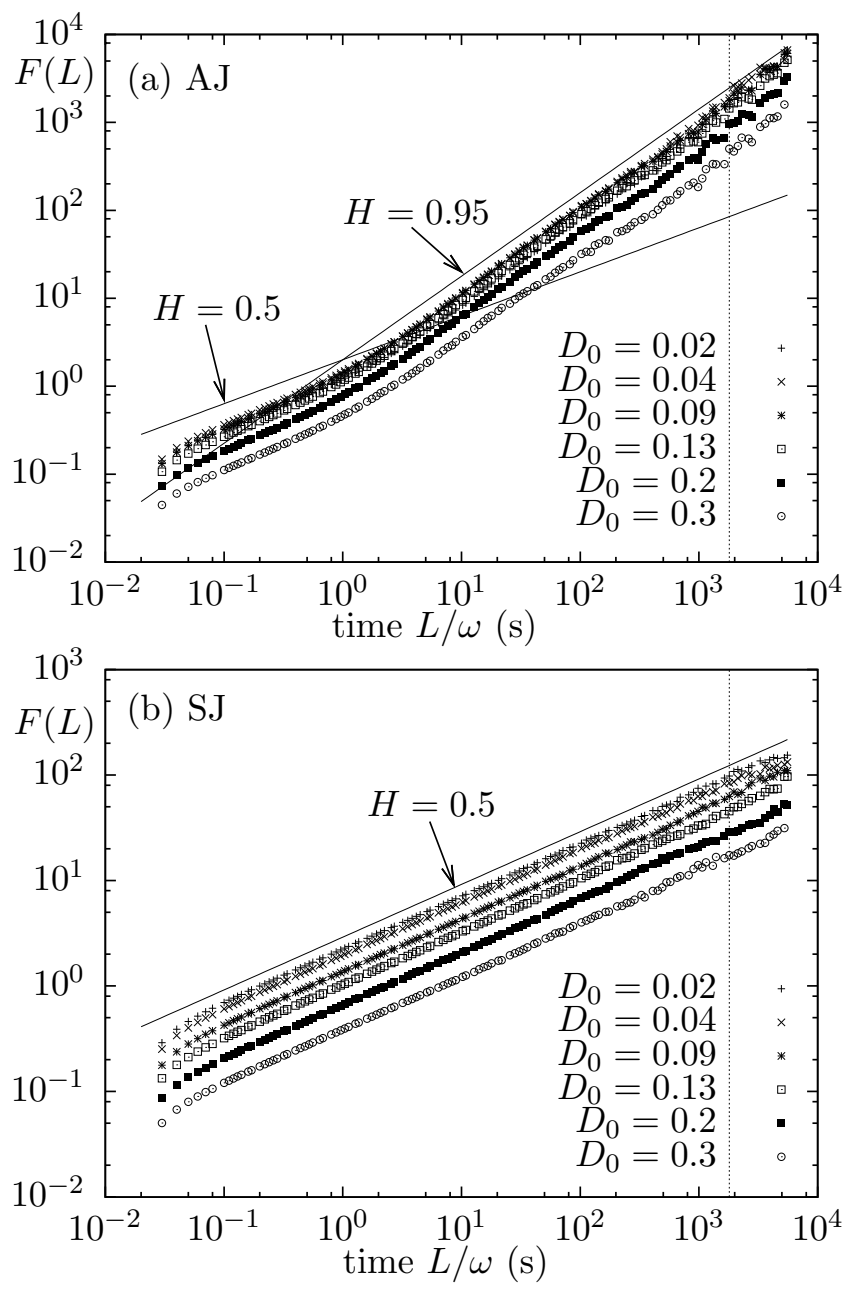

Fig. 7. Detrended Fluctuation Analysis applied to random walks driven by events derived from $U_{x}$ velocity component. (a) AJ rule. (b) SJ rule. The results for different thresholds $D_{0}$ are reported. The continuous lines are reported as an eye-guide. The vertical dotted line corresponds to the averaging time $T=1800 \mathrm{~s}$.

$z=H y+C$,

where $C$ is a constant.

In Fig. 7 we reported the DFA function $F(L)$ in log-log plots, for the two walking rules (AJ and $\mathrm{SJ}$ ) and for different thresholds. The slope of both AJ and SJ curves in the $\log -\log$ plot are approximately independent of the threshold value. This was true for the threshold values adopted, while remarkably different values are found for lower and larger values. Incidentally, we found that for extremely low threshold values the definition of event was dominated by erratic fluctuations, yielding a significantly smaller $H$ for the AJ rule, related to the detection of spurious events. For large threshold values the number of events was too small for our statistical analysis. The effect of the threshold is seen only in the intercept of the curves or, equivalently, in the area below the curve $F(L)$ : smaller threshold values correspond 
to greater number of events and, then, to greater $F(L)$ (top curves). The presence of a short-range or white noise is revealed by the short-time behavior of the AJ rule (Fig. 7a). In fact, the thresholding procedure detects not only genuinely complex Non-Poisson events but also spurious events that, following Allegrini et al. (2010b), we can assume as Poisson events. Thus, a superposition of a Non-Poisson and a Poisson point process would yield, for the second moment of the AJ rule, the sum of the second moments stemming from the two independent processes:

$\sigma^{2}(t)=P_{\mathrm{p}} \sigma_{\mathrm{p}}^{2}(t)+P_{\mathrm{n}} \sigma_{\mathrm{n}}^{2}(t)$,

where $P_{\mathrm{p}}$ and $P_{\mathrm{n}}$ are the statistical weights, $\sigma_{\mathrm{p}}$ and $\sigma_{\mathrm{n}}$ the standard deviations, for the Poisson and Non-Poisson processes, respectively. Our data suggest that the ratio $P_{\mathrm{p}} / P_{\mathrm{n}}$ decreases as the threshold increases. This takes into account the shift towards the left, upon threshold increase, of the knee marking the transition from normal diffusion to superdiffusion in the AJ rule (see Fig. 7a).

In Table 2 the estimated values of the diffusive scaling $H$ of the AJ walking rule are reported for each velocity component. The $H$ values are estimated by averaging over the different thresholds. For each threshold, the fitted values are derived in the interval going from about $5 \mathrm{~s}$ to $500 \mathrm{~s}$, where the scaling behavior is more evident in the AJ rule. A second fit was done up to $1000 \mathrm{~s}$ without significant changes. As said above, the AJ rule does not allow to discriminate among the ranges $\mu<2$ and $\mu>2$. However, the normal scaling $H \simeq 1 / 2$, found in the $\mathrm{SJ}$ rule, restricts the range to the interval $\mu \geq 2$. As a matter of fact, even in the case $\mu<2$ the superimposed Poisson noise would yield a normal scaling $H=1 / 2$ in the SJ rule. However, notice that Eq. (22) holds also for the $\mathrm{SJ}$ rule and we expect a sub-diffusive behavior in the short-time region with a knee shifting towards the right upon threshold increase. However, there is no evidence of a knee in the SJ rule (see Fig. 7b) and this suggests both terms of Eq. (22) yield normal diffusion. Another clue is given by the fractal dimension analysis shown in Fig. 3b, which gives $D_{\mathrm{F}}=1$ for all threshold values. As shown in Fig. 3a, this is another strong indication that $\mu>2$. Notice that, for the reasons discussed above and later in the conclusions, we cannot use the direct inspection of the WT distribution to prove that $\mu>2$, as we cannot assume that the diffusion process is strictly related to the WT distributions displayed in Fig. 4. As a counter-example, we refer to the superposition of Poisson and Non-Poisson point processes described above, a condition in which reliable information cannot be extracted directly from the WT distribution and the diffusion scaling is related to a "hidden" Non-Poisson renewal process (Allegrini et al., 2010b).

Inverting Eq. (17), which is based on the renewal hypothesis, we find the values of the complexity index $\mu_{D}$ reported in the last column of Table 2. The label $D$ is used to distinguish this complexity index and those evaluated from the WT distribution and to underline that it is computed from the
Table 2. Diffusion scaling $H$ of the AJ rule and complexity index $\mu_{D}$ for each velocity component. The values of $\mu_{D}$ are here derived from the diffusion scaling $H$ under the renewal assumption, i.e. by inverting Eq. (17). The average and the range of fitted $(H)$ and computed $\left(\mu_{D}\right)$ values are reported.

\begin{tabular}{ccc}
\hline $\begin{array}{c}\text { velocity } \\
\text { component }\end{array}$ & $H(\mathrm{AJ})$ & $\mu_{D}$ \\
\hline$u$ & $0.95(0.93-0.96)$ & $2.1(2.08-2.14)$ \\
$v$ & $0.95(0.93-0.96)$ & $2.1(2.08-2.14)$ \\
$w$ & $0.97(0.95-0.98)$ & $2.06(2.04-2.1)$ \\
\hline
\end{tabular}

diffusion scaling. The estimated values of $\mu_{D}$ are slightly above 2. This is a critical value marking the transition from a finite to an infinite mean WT and, then, between a slow relaxation to an asymptotic equilibrium state $(2<\mu<3)$ and no relaxation at all $(1<\mu \leq 2)$.

\subsection{Effect of data detrending and normalization on diffusion scaling}

We give a brief account of the robustness of the diffusion scaling analysis considering different definitions of the fluctuating signal, i.e. we substitute the definition given in Eq. (3) with two alternative ones. In the first one we evaluate the mean wind with a linear detrending procedure. Then, the fluctuation is:

$\widetilde{S}_{1}(t)=\frac{S_{1}(t)-[a(k) t+b(k)]}{\sigma_{1}(k)}$,

where, analogously to Eq. (3), $k$ labels the half-hour containing the time $t, \bar{S}_{k}(t)=a(k) t+b(k)$ is the linear trend evaluated with a least-squares straight line fit over nonoverlapping time windows of $30 \mathrm{~min}$ (the half-hour $k$ ) and $\sigma_{1}(k)$ is the analogous of $\sigma(k)$ in Eq. (2). In the second alternative definition, we use again the Moving Average method, but without applying the normalization with $\sigma(k)$. Then, we get:

$\widetilde{S}_{2}(t)=S(t)-\bar{S}(t)=\sigma(k) \tilde{S}(t)$.

Note that the statistical moments of the fluctuation increments in this last definition correspond to the standard structure functions widely studied in turbulence and relating to the energy flux $\epsilon$ across the different scales of turbulence (Frish, 1995).

In Fig. 8 the DFA analysis for events extracted from the fluctuations defined with linear detrending, Eq. (23), is shown. No dependence on the threshold is seen in the scaling behavior, which is very similar to that obtained applying the moving average method for both AJ and SJ rules. The fitted values confirm these qualitative observations. In Fig. 9 the normalized fluctuations, Eq. (3), and not normalized fluctuations, Eq. (24), are compared. Even in this case, no evident effect of the fluctuation definition is seen on the scaling 

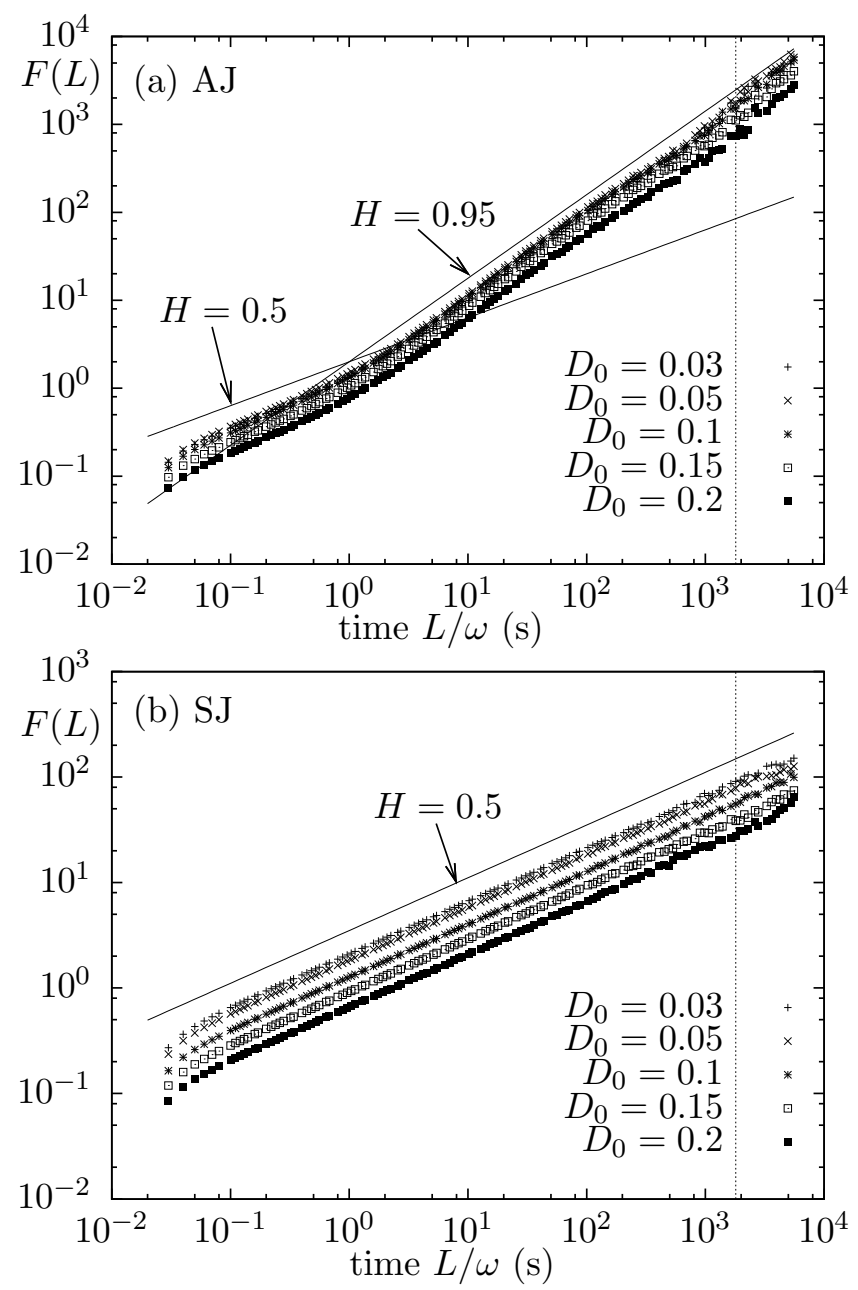

Fig. 8. Same as Fig. 7, but with events extracted from the fluctuating signal defined in Eq. (23).

behavior, at least on time below the averaging time, i.e. below 30 min (see vertical line in Figs. 7, 8 and 9). Apart from scaling, some qualitative differences are seen, especially on times of order of $30 \mathrm{~min}$, but this is not a surprising effect. This is more evident in Fig. 9, where the not normalized case deviate by the normalized one at about $500 \mathrm{~s}$. It is worth noting that, even being not accurate over times of the order of $30 \mathrm{~min}$ or larger, the DFA scaling region seems to be slightly more extended in the normalized case than in the not normalized case, so that the deviations could be an effect of slow non-stationary trends associated with slow variations of the local turbulent intensity.

\section{Discussions and concluding remarks}

Self-organized coherent structures have been recognized to play a central role in turbulence, being the emergence of these highly correlated fluid regions associated with an

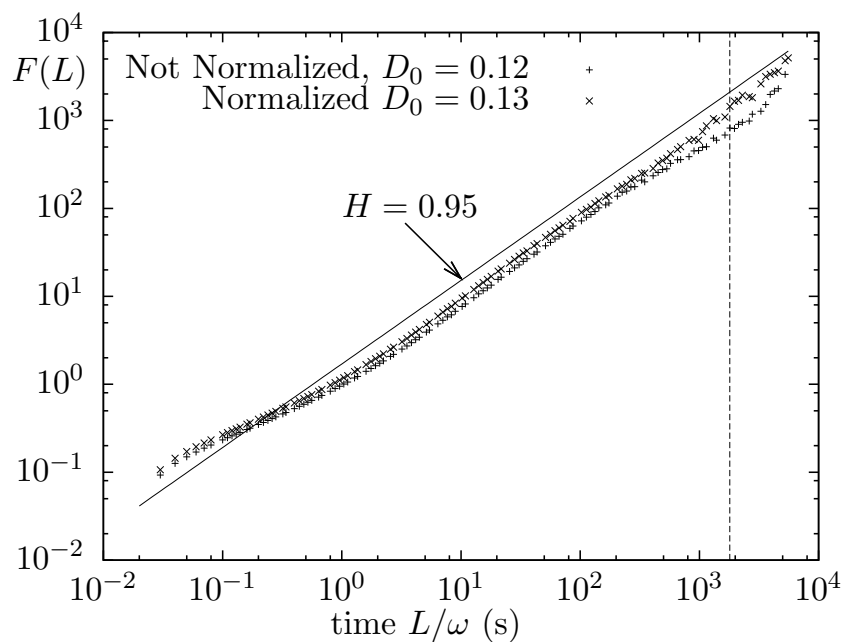

Fig. 9. DFA analysis: comparison of normalized and not normalized fluctuations, Eqs. (23) and (24)

enhancement of diffusion and transport (Mahrt, 1989; Finnigan, 2000). Diffusivity generated by coherent structures is a feature of particular interest from both theoretical and experimental point of view.

In this paper we investigated the time intermittent character of turbulent transport and we showed that it is driven by a self-similar point process leading to a well-defined diffusion scaling independent of the threshold used to define the intermittent transition events. We also showed that our analysis is robust under the change of the definition of turbulent fluctuations.

The diffusion scaling was found by means of a method based on event-driven random walks. The basic idea is that, as these random walks have a simple jump statistics, the diffusion depends only on the self-similarity and memory features of the event sequences, i.e. the complexity index $\mu$ and the inter-dependence among WTs. Further, in the case of renewal events and due to the simple jump statistics, analytical formulas relating diffusion scaling and complexity index are available. Notice that also the renewal assumption is crucial, but a direct correlation analysis on the WT sequences could lead to misleading results. As an example, when two or more renewal processes are superposed, the final global process would appear as a non-renewal point process (Allegrini et al., 2010b). However, as discussed in the introduction, the renewal assumption is reasonable and widely applied in the stochastic modeling of turbulent transport (Higbie, 1935; Perlmutter, 1961; Katul et al., 2006).

A direct evaluation of the complexity index $\mu$ from the WT distribution was also obtained. A strong dependence on the threshold was found with a $\mu$ range spanning from 1.7 to 4 . This is probably related to a combination of different effects: the multi-scaling nature of turbulence; the low statistics in the computation of the histogram, which is crucial in 
power-law fitting; the presence of spurious events related to the thresholding method, to real events not associated with self-organization or to both and which affect the shape of the WT distribution in a non-trivial way (Allegrini et al., 2010b).

The threshold dependence of $\mu$ evaluated from the WT distribution shows another important aspect of event-driven random walks, which essentially justifies their use in the analysis carried out in this paper. In fact, from the dependence $\mu=\mu\left(D_{0}\right)$ we expect a threshold dependence in the random walks and, then, in the diffusion scaling $H$. However, the AJ and SJ rule have the crucial property of adding linearly the contributions coming from independent point processes. This allows to the more rapid diffusion to emerge in the long-time regime. If enough data resolution is available and if the time scales of the different point processes are sufficiently far from each other, it it possible to detect also intermediate regimes. This can be seen in Figs. 7, 8 and 9, where a knee, dividing a short-time normal diffusion and a long-time super-diffusion, is evident.

It is important to note that, assuming renewal hypothesis, the diffusion scaling leads to a complexity index independent from the threshold. This complexity index $\mu_{D}$ is about 2.1 , definitively greater than 2 . The finding of $\mu_{D} \gtrsim 2$ is confirmed by the combination of the SJ rule with the fractal dimension analysis. In fact, it was numerically proved that the classical relation $\mu=1+D_{\mathrm{F}}$ is valid only for $\mu<2$, while $D_{\mathrm{F}}=1$ for $\mu>2$. It is interesting to note that, even when $D_{F}=1$, a self-similar behavior is still seen in the WT distribution and that this self-similarity in the WT distribution determines the scaling of event-driven diffusion in the $\mathrm{AJ}$ walking rule.

This observation leads us to conclude that an underlying process exists and that it drives the time intermittency of turbulent diffusion in the region of the ABL very near to the ground. The definition of event as fluid rapid acceleration leads us to identify this self-similar point process with the birth/death process of cooperation, which is associated with the emergence and decay of self-organized coherent structures. However, this interpretation is only hypothetical and needs further investigations.

The presence of coherent structures in turbulence determine the failure of the basic hypothesis of standard diffusion models, i.e. the separation between small and large scales, even when considering scales above the integral Eulerian scales (Stull, 1988). The scale separation hypothesis allows considering local turbulent fluxes, thus a local diffusivity can be defined. In some cases, even if fluxes are not local, an equivalent, but unphysical local diffusivity model can be used. Moreover, the emergence of counter-gradient phenomena, especially in convective conditions, makes this treatment inappropriate (Paradisi et al., 2001a,b). Diffusion scaling and intermittency are important features of transport in turbulent flows, particularly of diffusion processes related to coherent structures. Consequently, these features must be taken into account in the development of data-based stochastic models (Donner et al., 2009). In particular, the existence of a self-similar birth-death stochastic point process of cooperation driving the turbulent diffusion in the region of ABL near the ground has been assessed. This point process is perhaps related to the "external intermittency" claimed by Cava and Katul (2009) (see also Mahrt, 1989), but these aspects deserve further investigations and will be the subject of future work.

Acknowledgements. We thank the two referees for useful suggestions and discussion that greatly helped to improve our paper.

Edited by: S. Barbosa

Reviewed by: two anonymous referees

\section{References}

Akin, O. C., Paradisi, P., and Grigolini, P.: Periodic trend and fluctuations: the case of strong correlation, Physica A, 371, 157-170, 2006.

Akin, O. C., Paradisi, P., and Grigolini, P.: Perturbationinduced emergence of Poisson-like behavior in non-Poisson systems, J. Stat. Mech.-Theory E., P01013, doi:10.1088/17425468/2009/01/P01013, January 2009.

Allegrini, P., Barbi, F.,Grigolini, P., and Paradisi, P.: Renewal, modulation, and superstatistics in times series, Phys. Rev. E, 73, 046136, doi:10.1103/PhysRevE.73.046136, 2006.

Allegrini, P., Barbi, F., Grigolini, P., and Paradisi, P.: Aging and renewal events in sporadically modulated systems Chaos, Solitons and Fractals, 34, 11-18, 2007.

Allegrini, P., Bologna, M., Grigolini, P., and West, B. J.: Fluctuation-dissipation theorem for eventdominated processes, Phys. Rev. Lett., 99, 010603, doi:10.1103/PhysRevLett.99.010603, 2007.

Allegrini, P., Menicucci, D., Bedini, R., Fronzoni, L., Gemignani, A., Grigolini, P., West, B. J., and Paradisi, P.: Spontaneous brain activity as a source of ideal 1/f noise, Phys. Rev. E, 80, 061914, doi:10.1103/PhysRevE.80.061914, 2009a.

Allegrini, P., Bologna, M., Fronzoni, L., Grigolini, P., and Silvestri, L.: Experimental Quenching of Harmonic Stimuli: Universality of Linear Response Theory, Phys. Rev. Lett., 103, 030602, doi:10.1103/PhysRevLett.103.030602, 2009b.

Allegrini, P., Paradisi, P., Menicucci D., and Gemignani, A.: Fractal complexity in spontaneous EEG metastable-state transitions: new vistas on integrated neural dynamics Front, Physio., 1, 128, doi:10.3389/fphys.2010.00128, 2010a.

Allegrini, P., Menicucci, D., Bedini, R., Gemignani, A., and Paradisi, P.: Complex intermittency blurred by noise: Theory and application to neural dynamics, Phys. Rev. E, 82, 015103 doi:10.1103/PhysRevE.82.015103, 2010b.

Allegrini, P., Paradisi, P., Menicucci, D., Bedini, R., Gemignani, A., and Fronzoni, L.: Noisy cooperative intermittent processes: From blinking quantum dots to human consciousness, Journal of Physics: Conference Series, 306, 012027, doi:10.1088/17426596/306/1/012027, 2011.

Bershadskii, A., Niemela, J. J., Praskovsky, A., and Sreenivasan, K. R.: "Clusterization" and intermittency of temperature fluctuations in turbulent convection, Phys. Rev. E, 69, 056314, doi:10.1103/PhysRevE.69.056314, 2004a. 
Bershadskii, A., Niemela, J. J., and Sreenivasan, K. R.: Solar flares and thermal wind reversals: critical metastable states, Phys. Lett. A, 331, 15-19, 2004b.

Bianco, S., Grigolini, P., and Paradisi, P.: Fluorescence intermittency in blinking quantum dots: renewal or slow modulation?, J. Chem. Phys., 123, 174704, doi:10.1063/1.2102903, 2005.

Bianco, S., Grigolini, P., and Paradisi, P.: A fluctuating environment as a source of periodic modulation Chem. Phys. Lett., 438, 336340, 2007.

Cava, D. and Katul, G. G.: The effects of thermal stratification on clustering properties of canopy turbulence, Bound.-Lay. Meteorol., 130, 307-325, 2009.

Cox, D. R.: Renewal Theory, Methuen, London, 1962.

Donner, R., Barbosa, S., Kurths, J., and Marwan, N.: Understanding the Earth as a complex system - recent advances in data analysis and modelling in Earth sciences, Eur. Phys. J. Special Topics, 174, 1-9, 2009.

Feller, W.: An introduction to probability theory and its applications, 2nd Edn., Vol. II, Wiley, New York, 1971.

Finnigan, J.: Turbulence in plant canopies, Ann. Rev. Fluid Mech., 32, 519-571, 2000.

Frish, U.: Turbulence: the legacy of A. N. Kolmogorov, Cambridge University Press, Cambridge, 1995.

Gorenflo, R., Mainardi, F., Moretti, D., Pagnini, G., and Paradisi, P.: Fractional diffusion: probability distributions and random walk models, Physica A, 305, 106-112, 2002a.

Gorenflo, R., Mainardi, F., Moretti, D., and Paradisi, P.: Time fractional diffusion: a discrete random walk approach, Non Linear Dynam., 29, 129-143, 2002b.

Grigolini, P., Palatella, L., and Raffaelli, G.: Asymmetric anomalous diffusion: an efficient way to detect memory in time series, Fractals, 9, 439-449, 2001.

Grigolini, P., Leddon, D., and Scafetta, N.: Diffusion entropy and waiting time statistics of hard-X-ray solar flares Phys. Rev. E, 65, 046203, DOI: 10.1103/PhysRevE.65.046203, 2002.

Higbie, R.: The rate of absorption of a pure gas into a still liquid during short periods of exposure, Trans. Amer. Inst. Chem. Eng., 31, 365-388, 1935.

Holton, J. R.: An Introduction to Dynamic Meteorology, Academic Press, San Diego, 1992.

Kaimal, J. C. and Finnigan, J. J.: Atmospheric boundary layer flows: their structure and measurement, Oxford University Press, New York, 289 pp., 1994.

Katul, G. G., Kuhn, G., Schieldge, J., and Hsieh, C.: The ejectionsweep character of scalar fluxes in the unstable surface layer, Bound.-Lay. Meteorol., 83, 1-26, 1997.

Katul, G. G., Porporato, A., Cava, D., and Siqueira, M.: An analysis of intermittency, scaling, and surface renewal in atmospheric surface layer turbulence, Physica D, 215, 117-126, 2006.

Kenkre, V. M., Montroll, E. W., and Shlesinger, M. F.: Generalized Master Equations for Continuous-Time Random Walks, J. Stat. Phys., 9, 45-50, 1973.

Korabel, N., Klages, R., Chechkin, A. V., Sokolov, I. M., and Gonchar, V. Yu.: Fractal properties of anomalous diffusion in intermittent maps, Phys. Rev. E, 75, 036213, doi:10.1103/PhysRevE.75.036213, 2007.

Kubo, R., Toda, M., and Hashitsume, M.: Statistical Physics II, Nonequilibrium Statistical Mechanics, Springer, Berlin, 1985.

Lowen, S. B. and Teich, M. C.: Fractal renewal processes generate 1/f noise, Phys. Rev. E, 47, 992-1001, 1993.

Lowen, S. B. and Teich, M. C.: Fractal-based point processes, Wiley, New Jersey, 2005.

Mahrt, L.: Intermittency of atmospheric turbulence, J. Atmos. Sci., 46, 79-95, 1989.

Mahrt, L.: Variability and maintenance of turbulence in the very stable boundary layer, Bound.-Lay. Meteorol., 135, 1-18, 2010.

Mainardi, F. and Paradisi, P.: Fractional diffusive waves, J. Comp. Acoustics, 9, 1417-1436, 2001.

Manneville, P.: Intermittency, self-similarity and 1/f Spectrum in dissipative dynamical systems, Journal de Physique (France), 41, 1235-1243, 1980.

Metzler, R. and Klafter, J.: The random walk's guide to anomalous diffusion: a fractional dynamics approach Phys. Rep., 339, 1-77, 2000 .

Montroll, E. W.: Random walks on lattices, Proc. Symp. Appl. Math., Am. Math. Soc., 16, 193-220, 1964.

Narasimha, R. and Kailas, S. V.: Turbulent bursts in the atmosphere, Atmos. Environ., 24, 1635-1645, 1990.

Narasimha, R., Kumar, S. R., Prabhu, A., and Kailas, S. V.: Turbulent flux events in a nearly neutral atmospheric boundary layer, Phil. Trans. R. Soc. A, 365, 841-858, 2007.

Ott, E.: Chaos in dynamical systems, Cambridge University Press, Cambridge, UK, 2nd Edn., 2002.

Paradisi, P., Cesari, R., Mainardi, F., Maurizi, A., and Tampieri, F.: A generalized Fick's law to describe non-local transport effects, Phys. Chem. Earth, 26, 275-279, 2001a.

Paradisi, P., Cesari, R., Mainardi, F., and Tampieri, F.: The fractional Fick's law for non-local transport processes, Physica A, 293, 130-142, 2001b.

Paradisi, P., Allegrini, P., Barbi, F., Bianco, S., and Grigolini, P.: Renewal, modulation and Blinking Quantum Dots, AIP Conf. Proc., 800, 92-97, 2005.

Paradisi, P., Grigolini, P., Bianco, S. and Akin, O. C.: Renewal aging in non-homogeneous Poisson processes with periodic rate modulation, Int. J. Bifurcat. Chaos, 18, 2681-2691, 2008.

Paradisi, P., Cesari, R., Contini, D., Donateo, A., and Palatella, L.: Characterizing memory in atmospheric time series: an alternative approach based on renewal theory, Eur. Phys. J. Special Topics, 174, 207-218, 2009a.

Paradisi, P., Cesari, R., and Grigolini, P.: Superstatistics and renewal critical events, Cent. Eur. J. Phys., 7, 421-431, 2009 b.

Paw, U. K. T., Brunet, Y., Collineau, S., Shaw, R. H., Maitani, T., Qiu, J., and Hipps, L.: On coherent structures in turbulence above and within agricultural plant canopies, Agr. Forest. Meteorol., 61, 55-68, 1992.

Peng, C. K., Buldyrev, S. V., Havlin, S., Simons, M., Stanley, H. E., and Goldberger, A. L.: Mosaic organization of DNA nucleotides, Phys. Rev. E, 49, 1685-1689, 1994.

Perlmutter, D. D.: Surface renewal models in mass transfer, Chem. Eng. Sci., 16, 287-296, 1961.

Poggi, D. and Katul, G. G.: The ejection-sweep cycle over bare and forested gentle hills: a laboratory experiment, Bound.-Lay. Meteorol., 122, 493-515, 2007.

Poggi, D. and Katul, G. G.: Evaluation of the turbulent kinetic energy dissipation rate inside canopies by zero- and level-crossing density methods, Bound.-Lay. Meteorol., 136, 136-219, 2010.

Schertzer, D. and Lovejoy, S.: Physically based rain and cloud modeling by anisotropic, multiplicative turbulent cascades, J. Geo- 
phys. Res., 92, 9692-9714, 1987.

Schertzer, D., Lovejoy, S., and Hubert, P.: An introduction to stochastic multifractal fields, in: Mathematical problems in environmental science and engineering, edited by: Ern, A. and Weiping, L., Series in Contemporary Applied Mathematics, Higher Education Press, 4, 106-179, 2002.

Schmitt, F., Vannitsem, S., and Barbosa, A.: Modeling of rainfall time series using two-state renewal processes and multifractals, J. Geophys. Res., 103, 23181-23193, 1998.

Shlesinger, M. F.: Asymptotic solutions of continuous-time random walks, J. Stat. Phys., 10, 421-434, 1974.
Silvestri, L., Fronzoni, L., Grigolini, P., and Allegrini, P.: Eventdriven power-law relaxation in weak turbulence, Phys. Rev. Lett., 102, 014502, doi:10.1103/PhysRevLett.102.014502, 2009.

Sreenivasan, K. R. and Bershadskii, A.: Clustering Properties in Turbulent Signals, J. Stat. Phys., 125, 1145-1157, 2006.

Stull, R. B.: An introduction to boundary layer meteorology, Kluwer Academic Publishers, Dordrecht, The Netherlands, 1988.

Taylor, G. I.: Diffusion by continuous movements, Proc. London Math. Soc., 20, 196-212, 1921.

Weiss, G. H. and Rubin, R. J.: Random Walks: Theory and Selected Applications, Adv. Chem. Phys., 52, 363-505, 1983. 